\title{
Analysis of a model for the treatment of wastewater by the activated sludge process
}

\author{
S. D. Watt* H. S. Sidhu* M. I. Nelson ${ }^{\dagger}$
}

(Received 28 October 2005; revised 7 June 2006)

\begin{abstract}
We investigate a model for the treatment of wastewater in the activated sludge process. This process is based on the aeration of waste water with flocculating biological growth, followed by the separation of treated waste water from biological growth. Part of this growth is then wasted, and the remainder is returned to the system. The wastewater reactor is assumed to be well mixed, so the mathematical formulation for this process can be represented by a continuously stirred tank reactor with recycle. This system is analysed by combining steadystate analysis with path-following techniques. In practice, wastewater is treated by a sequence of tanks arranged in series. By considering
\end{abstract}

*School of Physical, Environmental and Mathematical Sciences, University of New South Wales at the Australian Defence Force Academy, Canberra, ACT 2600, AusTRALIA. mailto:simon_watt@bigfoot.com

${ }^{\dagger}$ School of Mathematics and Applied Statistics, University of Wollongong, Wollongong, NSW 2522, AustraliA

See http://anziamj.austms.org.au/V47EMAC2005/Watt for this article, (C) Austral. Mathematical Soc. 2006. Published June 14, 2006. ISSN 1446-8735 
the performance of one tank, our work here provides a benchmark for comparing the performances of multiple tanks.

\section{Contents}

1 Introduction

2 Model equations

2.1 Dimensional model . . . . . . . . . . . . . . . . . . C4

2.2 Dimensionless equations . . . . . . . . . . . C5

3 Behaviour of the system

3.1 Steady state analysis . . . . . . . . . . . . . . C8

3.2 Numerical results . . . . . . . . . . . . . . . . . . . C8

3.3 Analysis of the Hopf points . . . . . . . . . . C C12

4 Performance of a single reactor

5 Conclusions

C16

References

C17

\section{Introduction}

We consider wastewater treatment using an activated sludge process. Curds [1] provides a mathematical model of this process. Figure 1 shows a schematic diagram of the activated sludge process occurring in a well mixed single reactor. There are two types of bacteria and two types of protozoa present in the system which break down the incoming sewage.

In his original papers $[1,2]$, Curds investigated the model numerically 


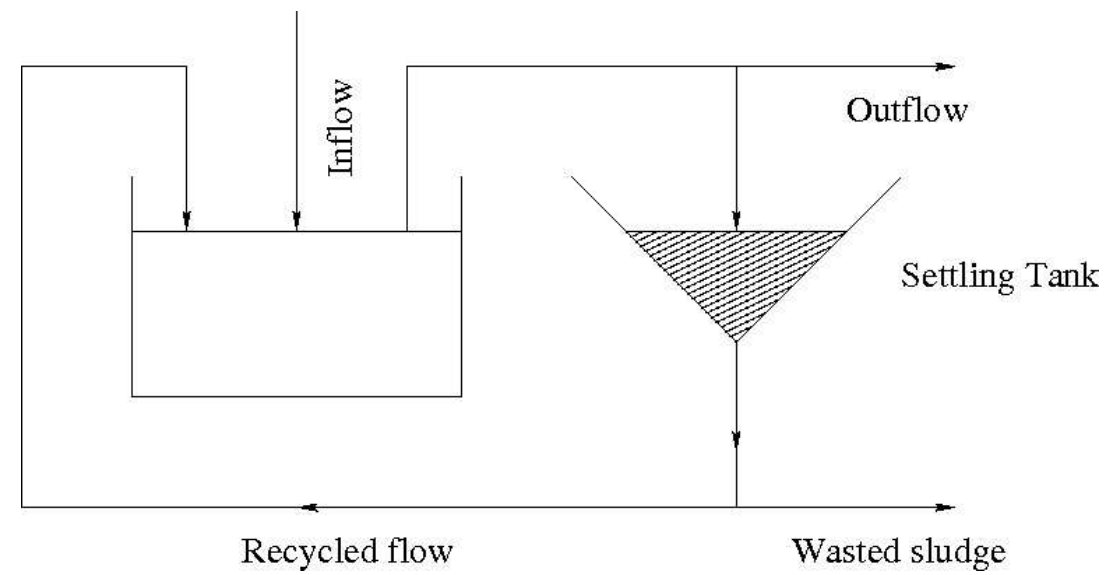

FigURE 1: A schematic diagram of the activated sludge process with a single reactor, a settling tank and recycling.

for only one set of physical parameters by numerically solving the system of ODEs. We investigate the model in greater detail by using bifurcation analysis, similar to that carried out in [3] through path following methods and algebraic results. We show that such methods enable optimal operating conditions to be readily obtained.

The main focus of this paper is to analyse the single tank case so that it serves as a benchmark when comparing results with multi-tank reactors. Curds [1] extended the system to include up to five reactors. This poses the question of what is the optimal number of reactors to improve the performance of the wastewater treatment process.

\section{Model equations}

To model the activated sludge treatment of wastewater, we use the system proposed by Curds [1] which consists of a well mixed single reactor. The 
sewage is pumped in at a constant rate, but then pumped out of the reactor at a constant, but higher, rate. What flows in then gets taken out of the system and the excess overflows into a settling tank, which concentrates the sludge bacteria and the attached protozoa. This then flows out of the settling tank and some of the sludge bacteria is wasted and the rest is recycled.

Apart from inflow and outflow of sewage and sludge, there are various biological reactions breaking down the sewage to bacteria and breaking down bacteria to protozoa, namely

$$
S+X \rightarrow X, \quad S+B \rightarrow B, \quad B+H \rightarrow H, \quad B+P \rightarrow P,
$$

where $S, X, B, H$ and $P$ are the concentrations of the substrate, sludge bacteria, sewage bacteria, free swimming protozoa and attached and crawling protozoa, respectively.

\subsection{Dimensional model}

The biochemical model here considers two types of bacteria: sludge bacteria and sewage bacteria and two types of ciliated protozoa: free-swimming ciliates and ciliates attached to sludge flocs. Together with concentration of the substrate in the tank, the activated sludge wastewater treatment process given in [1] is governed by a system of five ODEs:

$$
\begin{aligned}
V \frac{d S}{d t} & =F\left(S_{0}-S\right)-V X \frac{\mu_{X} S}{Y_{X}\left(K_{X}+S\right)}-V B \frac{\mu_{B} S}{Y_{B}\left(K_{B}+S\right)} \\
V \frac{d X}{d t} & =F\left(X_{0}-X\right)+a F(b X-X)+V X \frac{\mu_{X} S}{K_{X}+S} \\
V \frac{d B}{d t} & =F\left(B_{0}-B\right)+V B \frac{\mu_{B} S}{K_{B}+S}-V H \frac{\mu_{H} B}{Y_{H}\left(K_{H}+B\right)}-V P \frac{\mu_{P} B}{Y_{P}\left(K_{P}+B\right)}, \\
V \frac{d H}{d t} & =F\left(H_{0}-H\right)+V H \frac{\mu_{H} B}{K_{H}+B}
\end{aligned}
$$


$V \frac{d P}{d t}=F\left(P_{0}-P\right)+a F(b P-P)+V P \frac{\mu_{P} B}{K_{P}+B}$,

where $S_{0}, X_{0}, B_{0}, H_{0}$ and $P_{0}$ are the various concentrations in the inflow, $V$ is the volume of the reactor tank, $F$ is the rate of the inflow, $a$ is the recycling factor and $b$ is the concentration factor of the sludge and attached protozoa after passing through the settling tank. The biological reactions are assumed to follow Michaelis-Menton dynamics [4], where $\mu_{i}$ is the maximum specific growth rate, $Y_{i}$ is the yield coefficient and $K_{i}$ is saturation constant for the various bacteria and protozoa $i=X, B, H, P$.

\subsection{Dimensionless equations}

By introducing dimensionless variables for the substrate concentration $\left(S^{*}=\right.$ $\left.S / K_{X}\right)$, the sludge bacteria concentration $\left(X^{*}=X /\left(Y_{X} K_{X}\right)\right)$, the sewage concentration $\left(B^{*}=B / K_{X}\right)$, the free-swimming protozoa $\left(H^{*}=H /\left(K_{X} Y_{H}\right)\right)$, the attached and crawling protozoa $\left(P^{*}=P /\left(K_{X} Y_{P}\right)\right)$ and time $\left(t^{*}=\mu_{X} t\right)$, the model in dimensionless form is

$$
\begin{aligned}
\frac{d S^{*}}{d t^{*}} & =\frac{1}{\tau^{*}}\left(S_{0}^{*}-S^{*}\right)-\frac{S^{*} X^{*}}{1+S^{*}}-\frac{\mu_{b}^{*}}{Y_{B}} \cdot \frac{B^{*} S^{*}}{K_{B}^{*}+S^{*}}, \\
\frac{d X^{*}}{d t^{*}} & =\frac{1}{\tau^{*}}\left(X_{0}^{*}-X^{*}\right)+\frac{a(b-1)}{\tau^{*}} X^{*}+\frac{S^{*} X^{*}}{1+S^{*}}, \\
\frac{d B^{*}}{d t^{*}} & =\frac{1}{\tau^{*}}\left(B_{0}^{*}-B^{*}\right)+\frac{\mu_{B}^{*} S^{*} B^{*}}{K_{B}^{*}+S^{*}}-\frac{\mu_{H}^{*} B^{*} H^{*}}{K_{H}^{*}+B^{*}}-\frac{\mu_{P}^{*} B^{*} P^{*}}{K_{P}^{*}+B^{*}}, \\
\frac{d H^{*}}{d t^{*}} & =\frac{1}{\tau^{*}}\left(H_{0}^{*}-H^{*}\right)+\frac{\mu_{H}^{*} B^{*} H^{*}}{K_{H}^{*}+B^{*}}, \\
\frac{d P^{*}}{d t^{*}} & =\frac{1}{\tau^{*}}\left(P_{0}^{*}-P^{*}\right)+\frac{a(b-1)}{\tau^{*}} P^{*}+\frac{\mu_{P}^{*} B^{*} P^{*}}{K_{P}^{*}+B^{*}},
\end{aligned}
$$

where $\tau^{*}=\left(\mu_{X} V / F\right)$ is the dimensionless residence time.

The dimensionless model contains eight experimentally controllable parameters $\left(S_{0}^{*}, X_{0}^{*}, B_{0}^{*}, H_{0}^{*}, P_{0}^{*}, \tau^{*}, a\right.$ and $\left.b\right)$ and seven biochemical kinetic 
constants $\left(K_{B}^{*}, K_{H}^{*}, K_{P}^{*}, Y_{b}, \mu_{B}^{*}, \mu_{H}^{*}\right.$ and $\left.\mu_{P}^{*}\right)$. Note that the constants $a$ and $b$ only ever appear in the combination $a(b-1)$, reducing the number of experimentally controllable constants to seven. The values for the kinetic constants are determined by the choice of microbial system. We take the dimensionless residence time $\left(\tau^{*}\right)$ as the primary bifurcation parameter, leaving the dimensionless substrate concentration in the feed $\left(S_{0}^{*}\right)$ and the dimensionless sewage bacteria in the feed $\left(B_{0}^{*}\right)$ as possible secondary bifurcation parameters.

A feature of this dimensionless scheme is that there is a one-to-one relationship between the dimensionless variables and their dimensional counterpart. Hence we will often write the dimensionless residence time as just the residence time and so on for other quantities.

\section{Behaviour of the system}

There are two classes of behaviour in the system (1)-(5): steady state (Figure $2 \mathrm{a}$ ) and periodic solution (Figure 2b). Both of these have been noted in $[1,2]$. As mentioned in [5], "the periodic operation of chemical reactors can lead to improved reactor performance by increasing more reaction products or a more valuable product distribution than a steady-state reactor operation." We verify if this occurs for the one reactor system studied here. Following [1], we choose the biochemical parameters in Table 1. We also define the inflow concentrations as $S_{0}=200 \mathrm{mg} / \mathrm{l}, X_{0}=0 \mathrm{mg} / \mathrm{l}$ (sterile feed), $B_{0}=30 \mathrm{mg} / \mathrm{l}, H_{0}=P_{0}=0 \mathrm{mg} / 1$ (no protozoa in the inflow), Recycle rate of $100 \%(a=1)$ and Concentration Factor of $1.9(b=1.9)$. These give the dimensionless parameters $S_{0}^{*}=40 / 3, X_{0}^{*}=H_{0}^{*}=P_{0}^{*}=0, B_{0}^{*}=2$, $K_{B}^{*}=2 / 3, K_{H}^{*}=K_{P}^{*}=4 / 5, \mu_{B}^{*}=5 / 3$ and $\mu_{H}^{*}=\mu_{P}^{*}=7 / 6$. The dimensional parameter given above were also used in [5], so we believe they are representative of realistic values. 


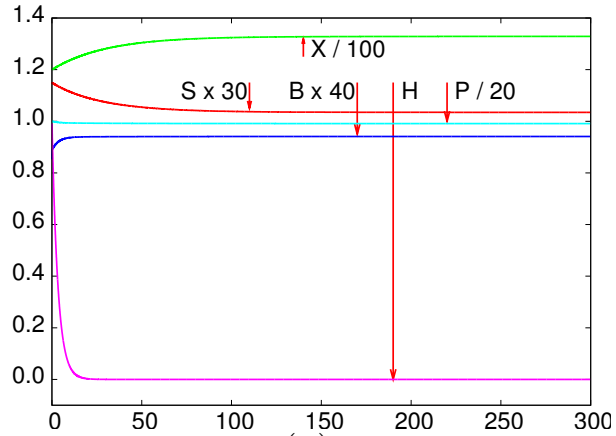

(a)

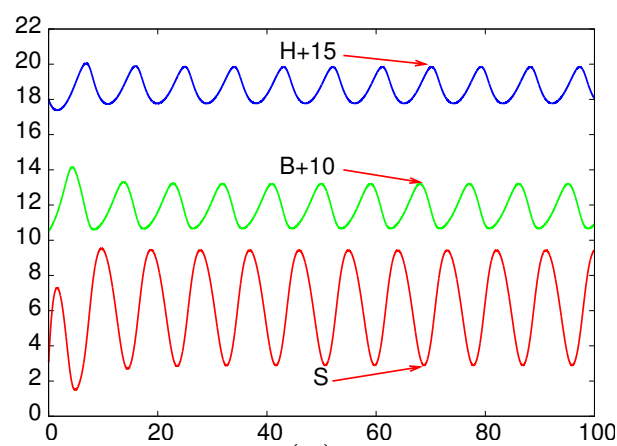

(b)

Figure 2: A numerical solution of (1)-(5) for (a) $\tau^{*}=3$ and (b) $\tau^{*}=1.3$. Note that the solutions have been scaled and translated to fit on the same graph.

TABLE 1: Biochemical parameters

Organism Maximum specific Saturation Yield

$i \quad$ growth rate $\mu_{i}\left(\mathrm{~h}^{-1}\right) \quad$ constant $K_{i}(\mathrm{mg} / \mathrm{l})$ coefficient $Y_{i}$

$\begin{array}{cccc}X & 0.3 & 15 & 0.5 \\ B & 0.5 & 10 & 0.5 \\ H & 0.35 & 12 & 0.5 \\ P & 0.35 & 12 & 0.5\end{array}$


TABLE 2: steady states for $\tau^{*}=3$.

\begin{tabular}{|c|ccccc|}
\hline Case & $S^{*}$ & $X^{*}$ & $B^{*}$ & $H^{*}$ & $P^{*}$ \\
\hline 1 & $\frac{111-\sqrt{12001}}{12}$ & 0 & $\frac{97+\sqrt{12001}}{24}$ & 0 & 0 \\
2 & $\frac{1}{29}$ & $\frac{240010}{2001}$ & $\frac{61}{23}$ & 0 & 0 \\
3 & $\frac{317+\sqrt{123609}}{51}$ & 0 & $\frac{2}{85}$ & 0 & $\frac{2823-5 \sqrt{123609}}{51}$ \\
4 & $\frac{1}{29}$ & $\frac{11987650}{90219}$ & $\frac{2}{85}$ & 0 & $\frac{20556}{1037}$ \\
5 & $\frac{71+\sqrt{7041}}{15}$ & 0 & $\frac{8}{25}$ & $\frac{897-5 \sqrt{7041}}{150}$ & 0 \\
6 & $\frac{1}{29}$ & $\frac{697418}{5307}$ & $\frac{8}{25}$ & $\frac{2682}{1525}$ & 0 \\
\hline
\end{tabular}

\subsection{Steady state analysis}

The steady states of (1)-(5) are found by setting the time derivatives to equal zero. The resulting equations can be solved analytically. The solutions fall into six cases, each corresponding to different combinations of $X^{*}, H^{*}$ and $P^{*}$ equaling to zero. As an example, and as starting points for the path following methods, we provide the steady states for $\tau^{*}=3$ in Table 2 .

\subsection{Numerical results}

Although the steady-state solutions of (1)-(5) can be found analytically, we choose to find the solutions numerically using the path following software package AUTO [6], as it easily determines the stability of the solutions by finding the eigenvalues of the corresponding Jacobian and determines the location of Hopf bifurcations and branch points.

Figures 3-7 shows how the different cases of steady-solutions depend on the (dimensionless) residence time, $\tau^{*}$.

We note that the curves are monotonic, either increasing or decreasing, 


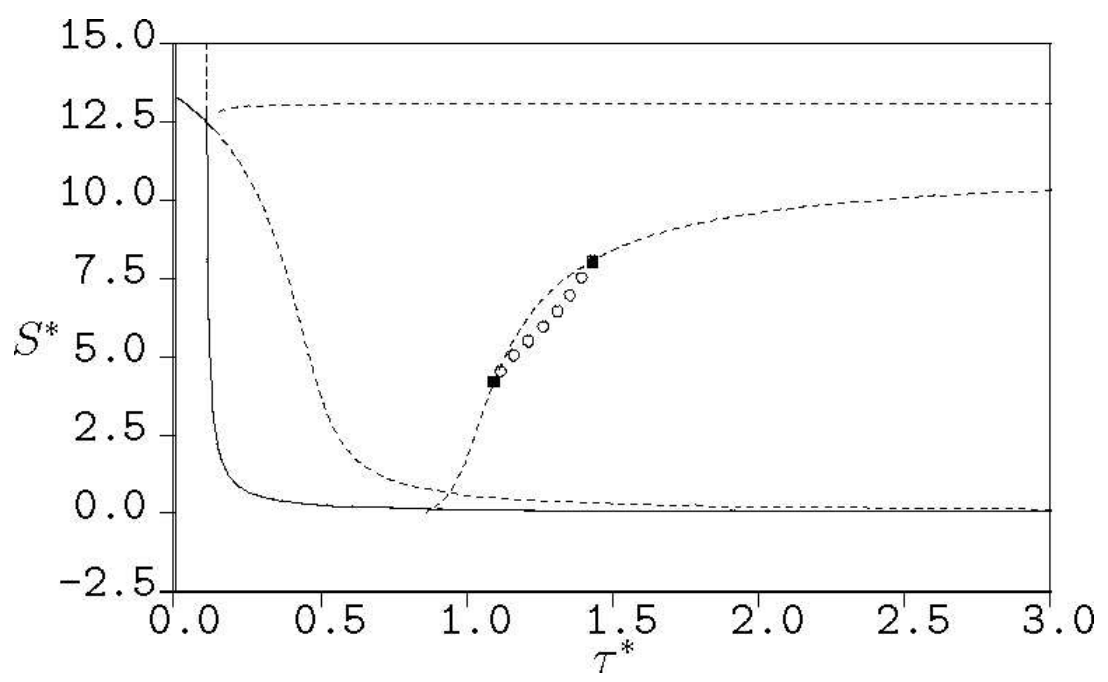

Figure 3: AUto shows the location of steady state solutions of (1)-(5) as functions of the residence time $\tau^{*}$ for $S^{*}, X^{*}, B^{*}, H^{*}$ and $P^{*}$ in Figures 3-7 respectively. Note: steady state solutions from the different cases can lie on top of each other. For example, here the steady state solutions for Cases 2, 4 and 6 lie on each other on the lowest curve. This explains why six distinct curves are not observed in each of Figures 3-7. 


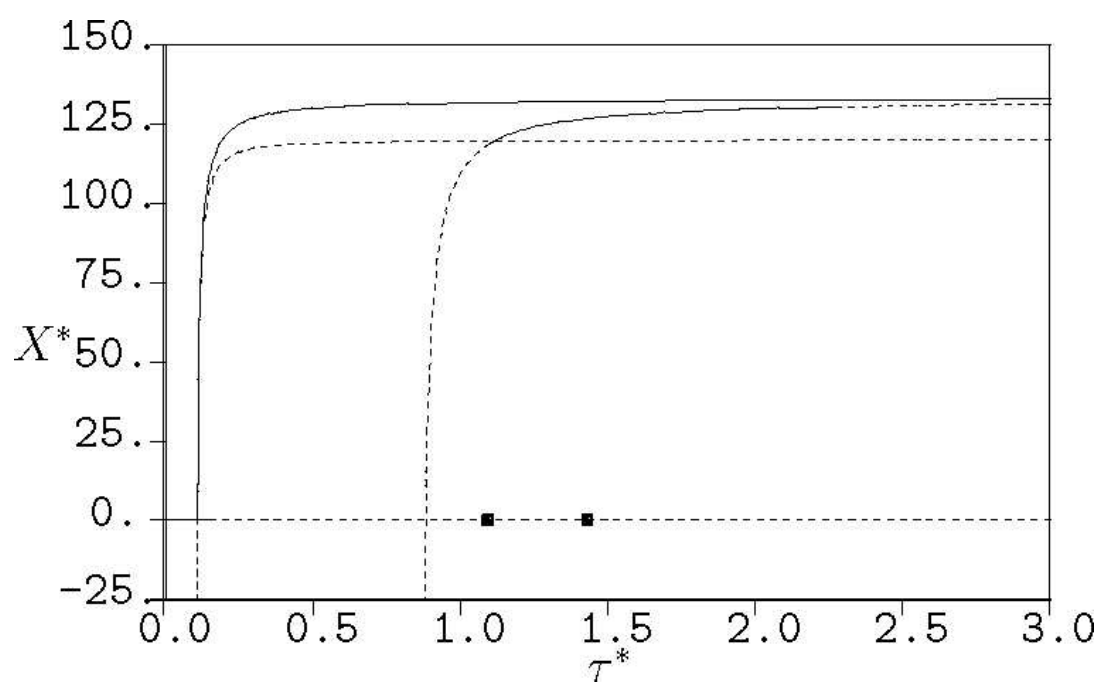

Figure 4: as for Figure 3.

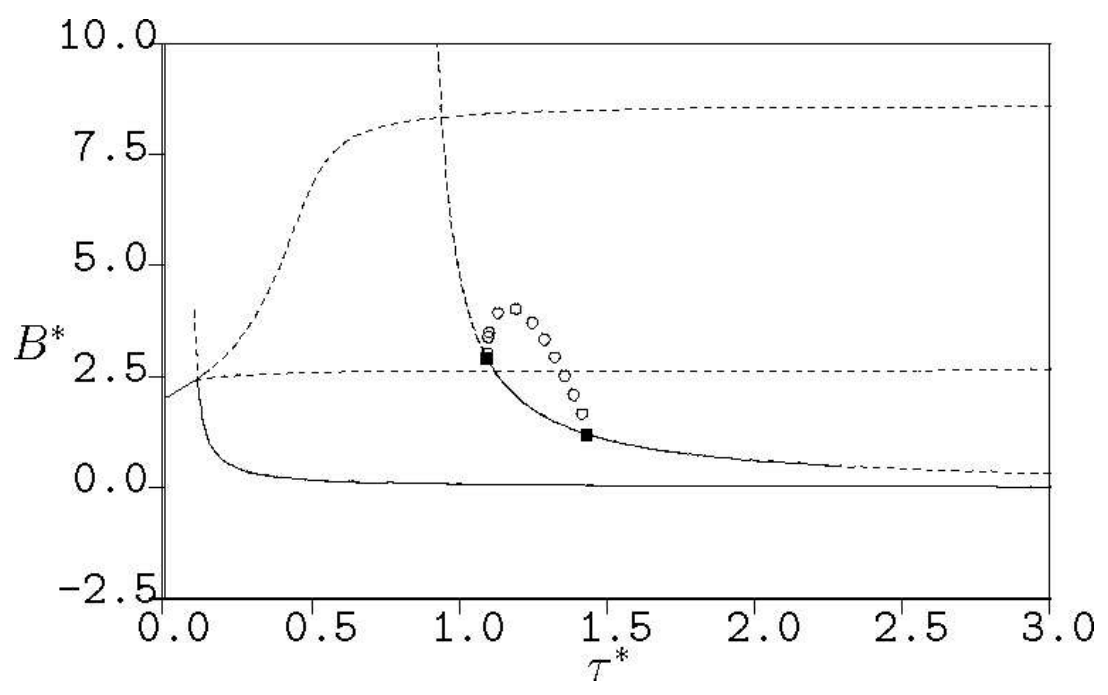

Figure 5: as for Figure 3. 


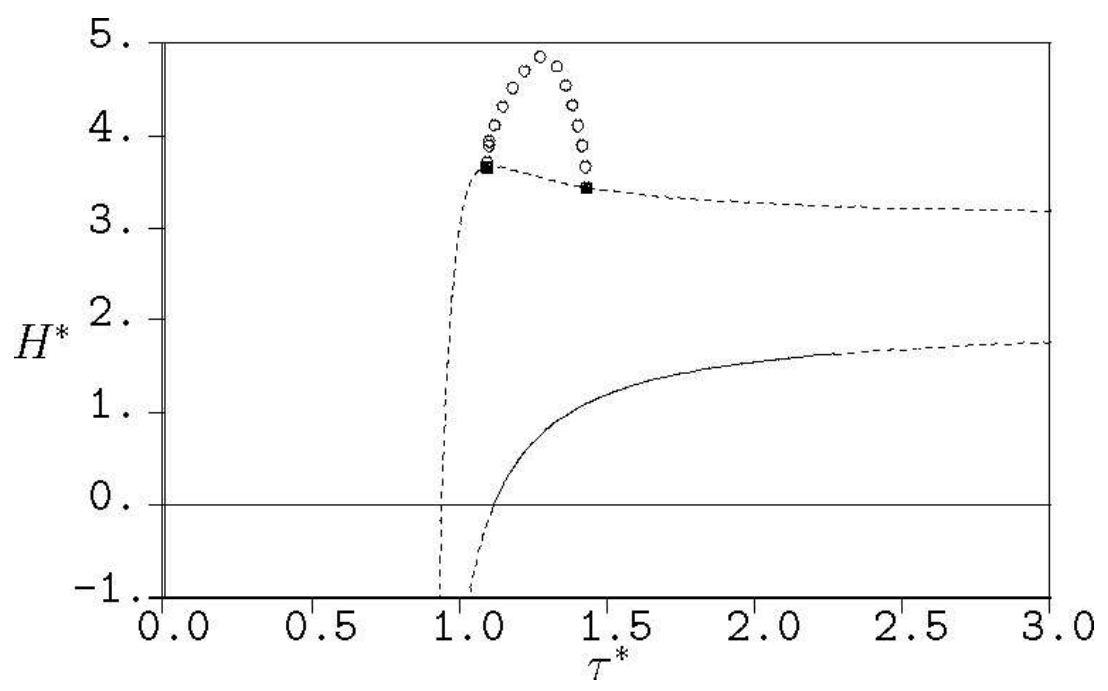

Figure 6: as for Figure 3.

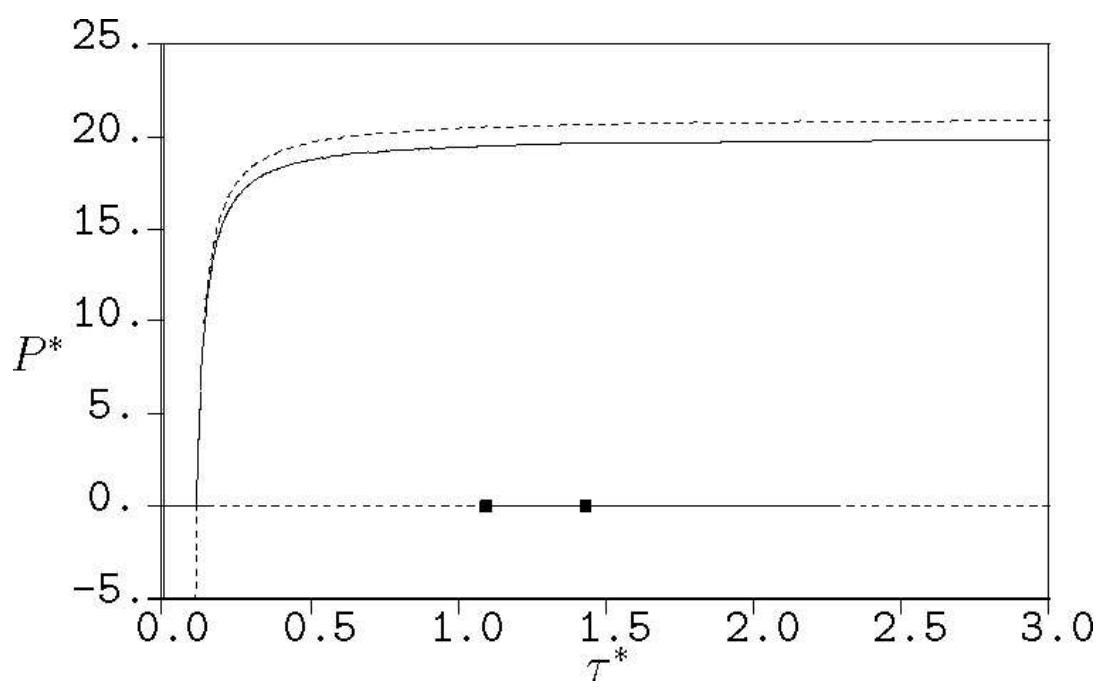

Figure 7: as for Figure 3. 
as a function of $\tau^{*}$. In the figures, the filled-in square boxes represent Hopf bifurcations, where the eigenvalues of the Jacobian matrix are purely imaginary, indicating the onset of the periodic solutions, and represented by open circles on the plots. We plotted the time average of the periodic solutions. Although the open circles imply that the orbits are unstable, by analysing the eigenvalues of the monodromy matrix, we find that the orbits are stable in the $S^{*}-B^{*}-H^{*}$-space (that is, choosing $X^{*}=P^{*}=0$ initially), as seen in Figure 2b.

\subsection{Analysis of the Hopf points}

Figures $3-7$ show that two Hopf points occur at $\tau^{*} \approx 1.09$ and $\tau^{*} \approx 1.42$. By varying one of the controllable parameters, the location of the Hopf points also changes.

In Figure 8a, by decreasing $S_{0}^{*}$ the two Hopf points move closer together, until they coalesce at a point (known as the double Hopf point or the $H 2_{1}$ degenerate point), after which there are no Hopf points, as shown in Figure 8b. Decreasing $B_{0}^{*}$, the Hopf points reappear again, as shown in Figure 8c. From Figure 8 and other numerical investigations, we note that the Hopf points only seem to occur on one branch of the steady state solution.

We can find an analytic relationship between the location of the two Hopf points in the $\left(\tau^{*}, S_{0}^{*}\right)$-parameter plane (see Figure 9a). The location of the $H 2_{1}$ point is $\left(\tau^{*}, S_{0}^{*}\right) \approx(1.28,10,59)$. Hence for $S_{0}^{*}<10.59$, no Hopf points will occur and therefore no oscillations can take place for any residence time or flow rate.

By repeating the above process for different values of $B_{0}^{*}$, we determine the $H 2_{1}$ locus in the $\left(S_{0}^{*}, B_{0}^{*}\right)$-parameter plane, as shown in Figure $9 \mathrm{~b}$. This divides the parameter space into two regions where oscillations are and are not possible for a range of residence times. 


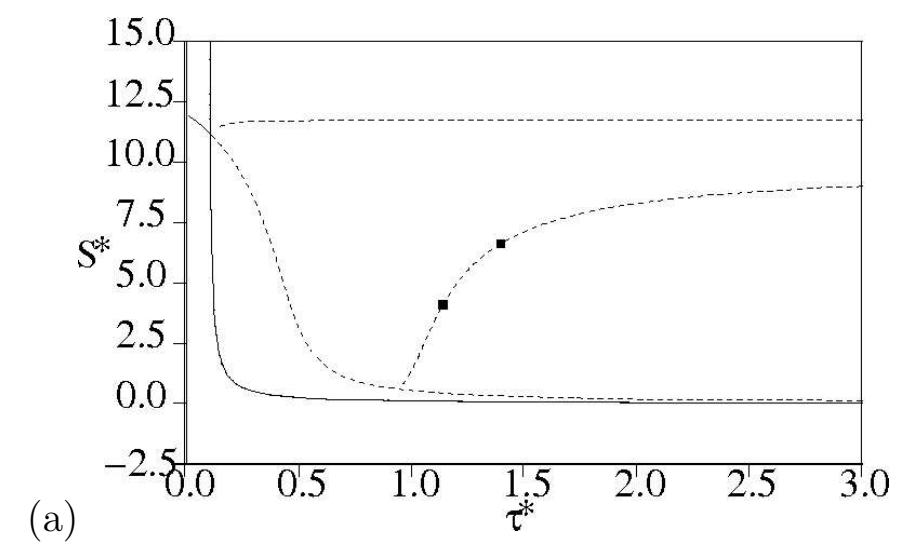

(a)

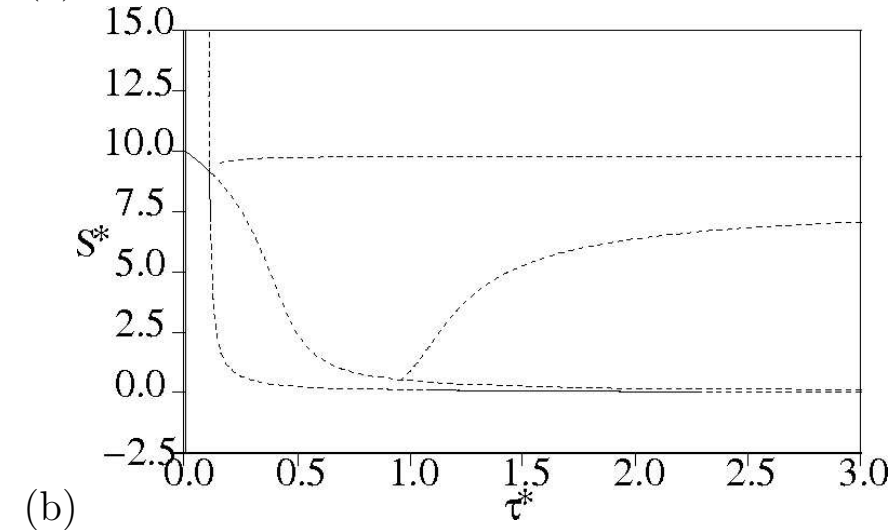

(b)

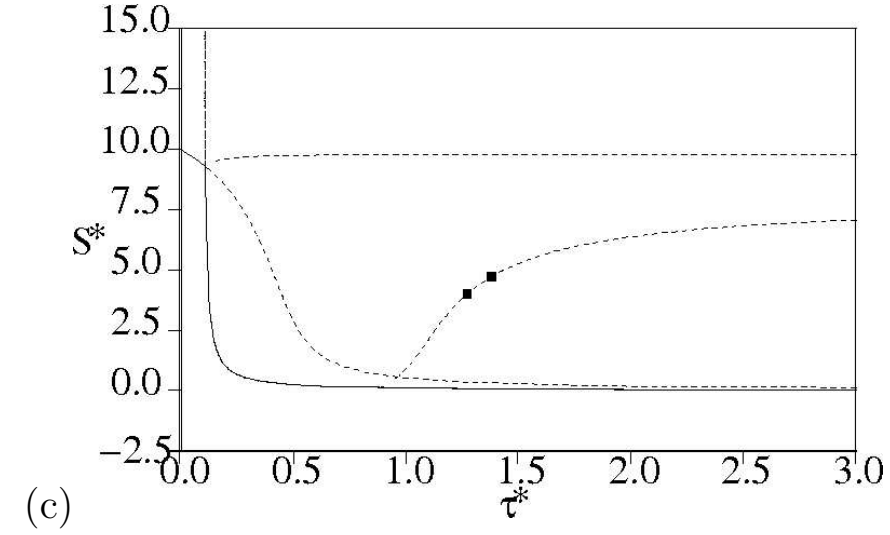

Figure 8: The location of the Hopf bifurcations for (a) $S_{0}^{*}=12$ and $B_{0}^{*}=2$; (b) $S_{0}^{*}=10$ and $B_{0}^{*}=2$; (c) $S_{0}^{*}=10$ and $B_{0}^{*}=1.7$. Note that the Hopf points are denoted by solid black boxes. 


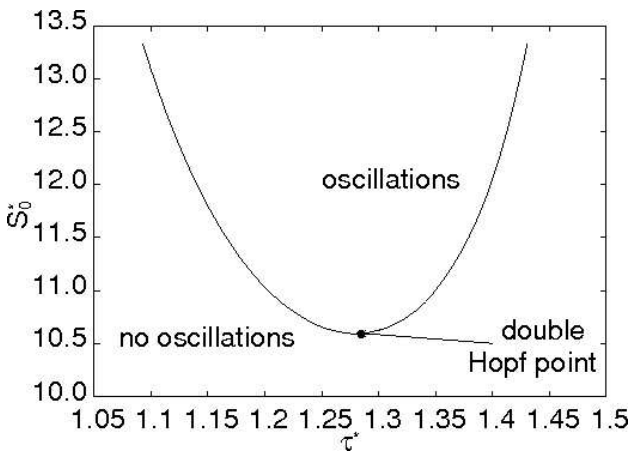

(a)

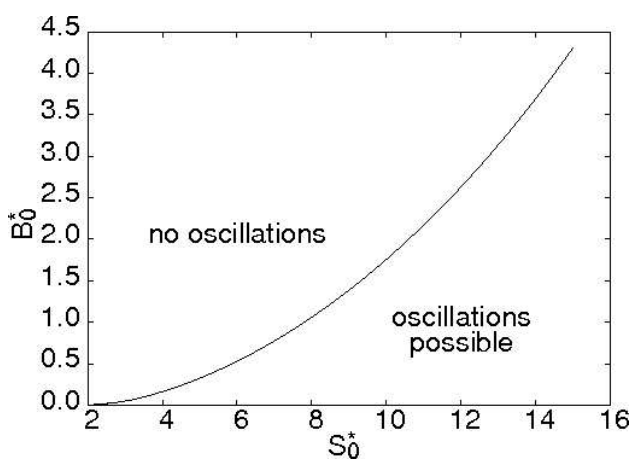

(b)

Figure 9: Graphs showing (a) the location of the Hopf locus for $B_{0}^{*}=2$ and (b) the two regions where oscillations are and are not possible in the $\left(S_{0}^{*}-B_{0}^{*}\right)$-parameter space.

\section{Performance of a single reactor}

Using the analysis from the previous sections, we now determine the conditions for optimising the performance for a single reactor. The definition of reactor performance is very much dependent upon the process involved. For example, Sidhu and Nelson [3] used the reactor productivity, $X^{*} / \tau^{*}$, as a performance indicator. Here we choose the treatment efficiency, proposed in [7]:

$$
\text { Treatment Efficiency }=100 \times\left(\frac{S_{0}^{*}-S^{*}}{S_{0}^{*}}\right) .
$$

The two extreme cases are: $S^{*}=S_{0}^{*}$ where none of the substrate has been broken down, so the efficiency is $0 \%$; and $S^{*}=0$ where all of the substrate has been broken down, so efficiency is $100 \%$. In Figure 10, we show the different efficiencies for the four different steady state substrate concentrations as a function of the residence time. Note that for $H^{*}=P^{*}=0$ (Case 2 from Section 3.1), $H^{*}=0$ (Case 4 ) and $P^{*}=0$ (Case 6), the steady state substrate concentrations are the same and so lie on top of each other, on curve $D$. For 


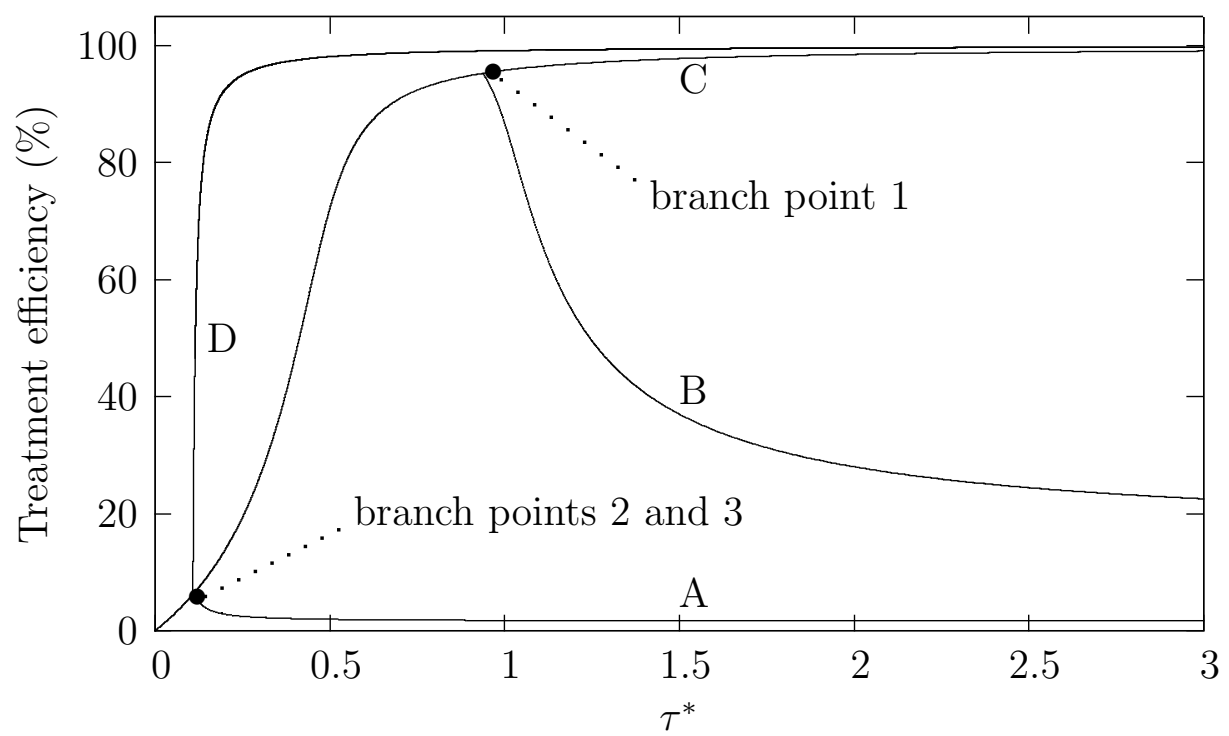

FiguRE 10: The treatment efficiency of a single reactor as a function of the residence time where: $A$ denotes $X^{*}=H^{*}=0 ; B$ denotes $X^{*}=P^{*}=0$; $C$ denotes $X^{*}=H^{*}=P^{*}=0$; and $D$ denotes $H^{*}=P^{*}=0, H^{*}=0$ and $P^{*}=0$. 
the chosen parameters, only two of the branches are globally stable: Case 1 for $\tau^{*}<0.114$ and Case 4 for $\tau^{*}>0.114$. When measuring the efficiency of the system, we only have to worry about these two cases. From the figure, one conclusion would be to make the residence time as long as possible, corresponding to slowing down the inflow rate or having a reactor with a very large volume. However, infinite residence times are not possible in practice. For a desired efficiency of $99 \%$, we need to have a residence time $\tau^{*}>0.85$ (that is, residence time longer than 2.83 hours). If we were interested in periodic solutions, as they exist for $1.09<\tau^{*}<1.42$ (between the two Hopf points from Figures 3-7), the efficiency ranges between $39 \%$ and $68 \%$, which is significantly lower than optimal and gives a different result than [5].

\section{Conclusions}

We undertook a preliminary investigation of the activated sludge process for wastewater treatment using a single reactor. The process is modelled using five ODEs, corresponding to the change in the various concentrations: substrate, sludge bacteria, sewage bacteria, free swimming cilliated protozoa and attached and crawling protozoa.

Using the path following software package AUTO [6], we carried out a steady state analysis. We showed that for different controllable parameters, namely the substrate and sewage bacterial concentrations in the inflow and the rate of inflow, it is possible to have either a steady state or periodic behaviour. Consequently, we also find operating regimes where periodic behaviour is not possible.

Finally, we investigated the conditions for increasing the performance of the system. We used the treatment efficiency as the performance indicator. We found that, in general, the longer the residence time, the better the efficiency. Furthermore, we found that, unlike the observations of [5], periodic operation did not improve the efficiency of this system (efficiency was sig- 
nificantly lower than the steady state operation) for the parameter values considered by Curds [1]. The analysis undertaken here will be used as a benchmark for further work, particularly when we compare the performance between the single reactor and multi-reactor cascade for the wastewater treatment system.

Acknowledgment: This work was supported by a grant from the Australian Research Council (DP0559177).

\section{References}

[1] C. R. Curds. Computer simulations of microbial population dynamics in the activated-sludge process. Water Research, 5:1049-1066, 1971. C2, C3, C4, C6, C17

[2] C. R. Curds. A computer-simulation study of predator-prey relationships in a single-stage continuous-culture system. Water Research, 5:793-812, 1971. C2, C6

[3] H. S. Sidhu and M. I. Nelson. Improving bioreactor performance: are two CSTBs always better than one? Proceedings of the 33rd Australian Chemical Engineering Conference: Chemeca 2005, Brisbane, 2005. C3, C14

[4] J. Monod. The growth of bacterial culture. Annual review of Microbiology, 3:371-394, 1949. C5

[5] S. Jianqiang and A. K. Ray. Performance improvement of activated sludge wastewater treatment by nonlinear natural oscillations. Chem. Eng. Technol., 23(12):1115-1122, 2000. C6, C16 
[6] E. J. Doedel, T. F. Fairgrieve, B. Sandstede, A. R. Champneys, Y. A. Kuznetsov and X. Wang. AUTO97: Continuation and bifurcation software for Ordinary Differential Equations (with Hom-Cont). ftp://ftp.cs.concordia.ca/pub/doedel/auto 1998. C8, C16

[7] A. E. Ghaly, S. S. Sadaka and A. Hazza'a. Kinetics of an intermittent-flow, continuous-mix anaerobic reactor. Energy Sources, 22:525-542, 2000. C14 